T. Ohsawa

Nagoya Math. J.

Vol. 137 (1995), 145-148

\title{
ADDENDUM TO "ON THE BERGMAN KERNEL OF HYPERCONVEX DOMAINS”, NAGOYA MATH. J. 129 (1993), 43-52
}

\section{TAKEO OHSAWA}

0. In [O-1] it was proved that for any bounded hyperconvex domain $D$ in $\mathbf{C}^{2}$ the Bergman kernel function $K(z, w)$ of $D$ satisfies

$$
\lim _{z \rightarrow \partial D} K(z, z)=\infty .
$$

In case $n=1$, this is due to a behavior of sublevel sets of the Green function. The general case then follows by the extendability of $L^{2}$ holomorphic functions.

1. After the author finished typing the manuscript of [O-1], H. Tanigawa suggested to him an alternative proof of the one variable case. Her argument consisted of an observation that the logarithmic capacity $c_{\beta}(z)$ of any bounded hyperconvex domain in $\mathbf{C}$ is exhaustive and an assertion that $K(z, z)$ is exhaustive whenever so is $c_{\beta}(z)$. Unfortunately, her proof of the latter statement was too difficult for the author to follow, and seemingly not to be published anywhere. Therefore, he decided to fix her idea by giving a straightforward proof to the following.

THEorem. There exists a constant $A \in[\pi, 750 \pi]$ such that, for any Riemann surface $S$ and for any local coordinate $z$ on $S, \sqrt{A K(z, z)} \geq c_{\beta}(z)$ holds.

2. N. Suita $[\mathrm{S}]$ conjectured that $\pi$ can be taken as the above $A$. In fact he showed that

$$
\sqrt{\pi K(z, z)}=c_{\beta}(z)
$$

if $S=\{z \in \mathbf{C}|| z \mid<1\}$, and that

$$
\sqrt{\pi K(z, z)}>c_{\beta}(z)
$$

if $S=\{z \in \mathbf{C}|r<| z \mid<1\}$ for some $r \in(0,1)$. The author hopes that our method may give a new insight into this subtle question.

Received November 19, 1993. 
3. Let $S$ be any Riemann surface, and let $z$ be any local coordinate of $S$ defined on a coordinate neighborhood, say $U$. If $S$ admits the Green function $g$, the logarithmic capacity $c_{\beta}(z)\left(=c_{\beta}(z(p))\right)$ is defined by

$$
-\log c_{\beta}(z(p))=\lim _{q \rightarrow p}(g(p, q)+\log |z(p)-z(q)|)
$$

for any $p \in U$. Otherwise we set $c_{\beta}(z) \equiv 0$. The Bergman kernel function $K(z)(=K(z, z))$ is defined by

$$
\log K(z)=\sup \log |Q(z)|^{2} .
$$

Here $Q$ runs through the set $\{Q \mid Q(z)$ is holomorphic on $U$ and there exists a holomorphic 1 -form $f$ on $S$ of $L^{2}$ norm 1 such that $\left.Q(z) \mathrm{d} z=f \mid U\right\}$.

4. For the proof of theorem we may assume that $c_{\beta}(z) \not \equiv 0$, since the result is trivial otherwise. For any point $p \in U$, we shall prove that there exists a holomorphic 1 -form $B_{p}$ on $S$ such that

$$
B_{p}\left|p=c_{\beta}(z) d z\right| p
$$

and

$$
\left\|B_{p}\right\|^{2} \leq 750 \pi
$$

Here $\|$ denotes the $L^{2}$ norm.

5. Let $\chi: R \rightarrow R$ be any $C^{\infty}$ function satisfying $\chi(t)=1$ on $(-\infty, 1]$, $\chi(t)=0$ on $(2, \infty)$ and $\left|\chi^{\prime}(t)\right|<2 \log 2$ everywhere. For simplicity we put

$$
g_{p}=g(p, \cdot) \text {. }
$$

Then we put

$$
f_{\varepsilon}= \begin{cases}\chi\left(\frac{-g_{p}-\log \varepsilon}{\log 2}\right) c_{\beta}(z(p)) & \text { on } U \\ 0 & \text { on } S \backslash U .\end{cases}
$$

Clearly, for sufficiently small $\varepsilon, f_{\varepsilon}$ is a $C^{\infty}$ function satisfying $f_{\varepsilon}(p)=c_{\beta}(z(p))$ and $\left\|f_{\varepsilon} d z\right\| \rightarrow 0$ as $\varepsilon \rightarrow 0$.

6. We assert that there exists a $c_{o}>0$ such that, for any $\varepsilon \in\left(0, c_{o}\right)$ one can find a square integrable $(1,0)$ form $\alpha_{\varepsilon}$ on $S$ satisfying

$$
\begin{gathered}
\bar{\partial} \alpha_{\varepsilon}=\bar{\partial} f_{\varepsilon} \wedge d z \\
\left.\left|\int_{U}\right| z\right|^{-2} \alpha_{\varepsilon} \wedge \bar{\alpha}_{\varepsilon} \mid<\infty
\end{gathered}
$$

and 


$$
\left\|\alpha_{\varepsilon}\right\|^{2}<750 \pi
$$

This suffices, since the required 1 -form $B_{p}$ will be obtained by putting $B_{p}=f_{\varepsilon} \wedge$ $d z-\alpha_{\varepsilon}$ for sufficiently small $\varepsilon$.

7. For that, givin $\varepsilon$ we look for a positive number $\delta$, a $C^{\infty}$ function $\rho$ : $S \rightarrow(0, \infty)$ and a conformal metric $d s^{2}$ on $S$ satisfying the following conditions (i) through (iii).

$$
i \delta \int_{U}|z|^{-2} \alpha \wedge \bar{\alpha}+i \int_{S} \alpha \wedge \bar{\alpha} \leq 5 i \int_{S} \rho \alpha \wedge \bar{\alpha}
$$

for any square integrable $(1,0)$ form $\alpha$ on $S$.

(ii) For any $C^{\infty}(1,1)$ form $\beta$ on $S \backslash\{p\}$ with supp $\beta \subset\left\{\log 2<g_{p}+\log \varepsilon<\right.$ $2 \log 2\}$, there exists a solution to $\bar{\partial} \alpha=\beta$ satisfying

$$
i \int_{S} \rho \alpha \wedge \bar{\alpha} \leq \int_{S} e^{2 g_{p}}|\beta|^{2} d v o l
$$

Here $|\beta|$ denotes the pointwise norm of $\beta$ and $d v o l$ denotes the volume form, both with respect to $d s^{2}$.

$$
\int_{S} e^{2 g_{p}}\left|\bar{\partial} f_{\varepsilon} \wedge d z\right|^{2} d v o l<150 \pi
$$

8. Obviously, we are through if there exist $\delta, \rho$ and $d s^{2}$ as above.

9. It is easy to see that (iii) is satisfied if we put

$$
d s^{2}=4 e^{-2 g_{p}} \varepsilon^{2}\left(e^{-2 g_{p}}+\varepsilon^{2}\right)^{-2} \partial g_{p} \bar{\partial} g_{p} .
$$

On the other hand, a general nonsense of elementary functional analysis tells us that (ii) is satisfied provided that there exists a $C^{\infty}$ positive function $\eta$ on $S$ such that,

$$
-i\left(\partial \bar{\partial} \eta+\eta^{-2} \partial \eta \wedge \bar{\partial} \eta\right) \geq 4 i \varepsilon^{2} e^{-2 g_{p}}\left(e^{-2 g_{p}}+\varepsilon^{2}\right)^{-2} \partial g_{p} \wedge \bar{\partial} g_{p}
$$

and

$$
\rho \leq e^{2 g_{p}}\left(\eta+\eta^{2}\right)^{-1}
$$

(cf. [O-2] Theorem 1.7).

10. Therefore our problem was reduced to finding $\rho$ and $\eta$ satisfying (4), (5) and (i) for some $\delta>0$.

11. For that, we put

$$
\eta=-\log \left(e^{-2\left(g_{p}+1\right)}+\varepsilon^{2}\right)+\log \left(-\log \left(e^{-2\left(g_{p}+1\right)}+\varepsilon^{2}\right)\right)
$$


for $\varepsilon \in\left(0, e^{-1}-e^{-2}\right)$. To simplify the computation, let

$$
\phi=\log \left(e^{-2\left(g_{p}+1\right)}+\varepsilon^{2}\right) .
$$

Then

$$
-\partial \bar{\partial} \eta=\partial \bar{\partial} \psi-\phi^{-1} \partial \bar{\partial} \psi+\phi^{-2} \partial \phi \wedge \bar{\partial} \psi
$$

and

$$
\eta^{-2} \partial \eta \wedge \bar{\partial} \eta=(\phi+\log (-\phi))^{-2}\left(1-\phi^{-1}\right)^{2} \partial \phi \wedge \bar{\partial} \psi
$$

Hence

$$
-i\left(\partial \bar{\partial} \eta+\eta^{-2} \partial \eta \wedge \bar{\partial} \eta\right) \geq i \partial \bar{\partial} \psi .
$$

But a straightforward computation shows that

$$
i \partial \bar{\partial} \phi \geq 4 i \varepsilon^{2}\left(e^{-2 g_{p}}+\varepsilon^{2}\right)^{-2} e^{-2 g_{p}} \partial g_{p} \bar{\partial} g_{p} .
$$

Thus (4) is satisfied by the above $\eta$. As $\rho$, we have only to put

$$
\rho=e^{2 g_{p}}\left(\eta+\eta^{2}\right)^{-1}
$$

In fact, since

$$
\begin{aligned}
& \sup _{t>0} e^{-2 t}\left(-\log \left(e^{-2(t+1)}+\varepsilon^{2}\right)+\log \left(-\log \left(e^{-2(t+1)}+\varepsilon^{2}\right)\right)\right) \\
\leq & \sup _{t>0} e^{2-2 T}(2 T+\log T+\log 2) \\
\leq & \sup _{t>0} e^{2-2 T}(3 T-1+\log 2) \\
< & \frac{3}{2} e<5
\end{aligned}
$$

one has (i) for sufficiently small $\delta$, in view of the behavior of $\rho$ near $p$.

\section{REFERENCES}

[O-1] Ohsawa, T., On the Bergman kernel of hyperconvex domains, Nagoya Math. J., 129 (1993), 43-52.

[O-2] - On the extension of $L^{2}$ holomorphic functions III: negligible weights, to appear in Math. $Z$.

[S] Suita, N., Capacities and kernels on Riemann surfaces, Arch. Rational Mech. Anal., 46 (1972), 212-217.

Department of Mathematics

Nagoya University

Nagoya, 464-01 Japan 